\title{
Recent advances in the structure-guided research and development of antimicrobial therapeutics
}

\author{
Dimitar Trifonov ${ }^{\ddagger}$, Tom Evers ${ }^{\ddagger}$ \\ ‡ National Research Tomsk State University, Tomsk, Russia
}

Corresponding author: Dimitar Trifonov (dtriffonoff@gmail.com)

Received: 09 Jul 2017 | Published: 17 Jul 2017

Citation: Trifonov D, Evers T (2017) Recent advances in the structure-guided research and development of antimicrobial therapeutics. BioDiscovery 20: e14952. https://doi.org/10.3897/biodiscovery.20.e14952

\begin{abstract}
Nowadays the research and development of novel antimicrobial therapeutics is failing to meet market needs. Clearly, the historical approach of empirical screens for antimicrobial drug discovery is not robust enough to solve the problem of antimicrobial resistance as fighting multidrug resistance pathogens (e.g. K. pneumonia, M. tuberculosis etc.) requires the identification of new drug targets and new classes of therapeutic compounds. However, it has been shown recently that structure-guided research and development can be a viable drug discovery strategy for antimicrobials in terms of determining novel targets and new drug classes. Here, we discuss recent advances and trends in the field related to contemporary techniques for structural elucidation of proteins (X-ray crystallography, nuclear magnetic resonance and cryo electron microscopy) as well as supporting computational tools. Specifically we highlight hardware improvements aimed at overcoming the limitations of the before-mentioned approaches for determining the $3 D$ structure of proteins. In terms of supporting software development the progress in image processing and virtual screening is brought up-to-date.
\end{abstract}

\section{Keywords}

Structure-guided research and development, antimicrobials, X-ray crystallography, nuclear magnetic resonance, cryo electron microscopy 


\section{Presenting author}

Dimitar Trifonov

\section{Presented at}

World BioDiscovery Congress 2017 